(c) American Dairy Science Association, 2004.

\title{
Development and Analysis of a Rumen Tissue Sampling Procedure
}

\author{
K. E. Lesmeister, P. R. Tozer, and A. J. Heinrichs \\ Department of Dairy and Animal Science \\ The Pennsylvania State University, University Park 16802
}

\section{ABSTRACT}

A procedure for rumen tissue sampling was developed to determine treatment effects on rumen development and papillae growth in young calves and to improve repeatability in rumen tissue sampling techniques. $\mathrm{Ru}-$ mens were collected from 42 male Holstein calves from 4 separate experiments. Rumen sampling areas $(n=9)$ included the caudal dorsal blind sac, cranial dorsal sac, cranial ventral sac, and the caudal and ventral portions of the caudal ventral blind sac. Right and left sides of the rumen were sampled. Five $1-\mathrm{cm}^{2}$ sections were removed from each area and measured for papillae length $(\mathrm{n}=$ 20/area), papillae width ( $n=20$ /area), rumen wall thickness $\left(\mathrm{n}=5\right.$ /area), and number of papillae per $\mathrm{cm}^{2}(\mathrm{n}=$ 5/area). Correlations between areas, samples, and measurements were obtained, and comparisons between experiments, areas, samples, and measurements were performed for all variables. In addition, power analyses were conducted for all variables to determine the efficacy of the procedure in detecting treatment differences. Results indicate that samples should be taken from the caudal and cranial sacs of the dorsal and ventral rumen to sufficiently represent papillae growth and development throughout the entire rumen. The procedure is capable of detecting treatment differences for papillae length and papillae width, has a decreased but acceptable capability of detecting treatment differences for rumen wall thickness, but appears limited in ability to detect treatment differences for papillae per square centimeter. In conclusion, rumen tissue sampling to determine extent of rumen development in calves can be performed in a nonbiased and repeatable manner utilizing a limited number of calves.

(Key words: rumen tissue sampling, papillae, correlation, power analysis)

Abbreviation key: A = caudal portion of caudal ventral blind sac, $\mathbf{L S N}=$ least significant number, $\mathbf{L B}=$ left side caudal dorsal sac, $\mathbf{L C}=$ left side cranial dorsal sac, $\mathbf{L D}=$ left side cranial ventral sac, $\mathbf{L E}=$ left side ventral portion

Received May 29, 2003.

Accepted October 23, 2003.

Corresponding author: A. J. Heinrichs; e-mail: ajh@psu.edu.

of caudal ventral blind sac, $\mathbf{P L}=$ papillae length, $\mathbf{P W}=$ papillae width, $\mathbf{P C}=$ papillae per square centimeter, $\mathbf{R B}=$ right side caudal dorsal sac, $\mathbf{R C}=$ right side cranial dorsal sac, $\mathbf{R D}=$ right side cranial ventral sac, $\mathbf{R E}=$ right side ventral portion of caudal ventral blind sac, RWT $=$ rumen wall thickness.

\section{INTRODUCTION}

The ruminal conditions necessary for papillae development have been previously elucidated (Brownlee, 1956; Flatt et al., 1958; Sutton et al., 1963). Alterations in dietary chemical and structural composition have influenced rumen papillae length (PL) and width (PW), rumen wall thickness (RWT), and papillae density (PC) (Brownlee, 1956; Harrison et al., 1960; Tamate et al., 1962). In addition, some have suggested that the rumen epithelium and ruminal muscle grow and develop independent of each other, indicating that dietary factors influencing one may not affect the other (Brownlee, 1956; Harrison et al., 1960). Continued research focusing on dietary manipulation to optimize the rate and extent of ruminal VFA production and development is still needed and could provide economic and health advantages to the dairy replacement industry. However, previous rumen sampling techniques have primarily obtained samples from the cranial ventral and caudal dorsal sacs of the rumen, corresponding to areas indicated to have the largest and most numerous papillae (Tamate et al., 1962; Nocek et al., 1984; Van Soest, 1994). Accounting for the importance of rumen papillae growth and the apparent within-rumen variation, development of a procedure that standardizes rumen tissue sampling, measurement, and analysis could greatly aid this field of research and fill a void in the available literature (Brownlee, 1956; McGavin and Morrill, 1976b). In addition, samples taken from multiple areas of the rumen may result in a more representative description of rumen growth and development. However, the possibility arises that some areas of the rumen and/or some measures of development may be better suited for determination of treatment differences than others, or that a single area of the rumen may be representative of multiple areas. The removal of highly variable areas from the analysis could also reduce procedural error, creating the possibility of de- 
creasing the number of observations per area and/or calves required per treatment. The objectives of this study were threefold, 1) to develop a repeatable and unbiased procedure for rumen tissue sampling, 2) to determine the areas of the rumen best suited for identifying treatment differences, and 3) to determine the proposed procedure's efficacy in rumen development research.

\section{MATERIALS AND METHODS}

\section{Rumen Tissue Sampling}

Reticulo-rumens were obtained from 42 male Holstein calves utilized in 4 separate experiments between 2000 and 2002, and representing 11 different dietary treatments. Experimental protocols and procedures were similar across experiments, with the exception of dietary treatments, weaning age, and age at slaughter. Briefly, all calves received $4 \mathrm{~L}$ of colostrum within $12 \mathrm{~h}$ of birth, and were fed a $20 \%$ all-milk protein and $20 \%$ fat milk replacer in 2 equal feedings, totaling $10 \%$ of birth weight until abrupt weaning ( 4 wk for 1 experiment and $5 \mathrm{wk}$ for 3 experiments). A calf starter of similar composition, with the exception of the dietary additives unique to each experiment, was offered ad libitum daily. Six calves from the first experiment conducted during the summer of 2000 were slaughtered at $6 \mathrm{wk}$ of age, and 6 calves from the second experiment conducted during the fall of 2000 and winter of 2001 were slaughtered at $5 \mathrm{wk}$ of age. Fifteen calves from the third experiment conducted through the winter of 2001 to the winter of 2002 were slaughtered at $4 \mathrm{wk}$ of age, and the final 15 calves from the fourth experiment conducted during the summer of 2002 were slaughtered at 5 wk of age. Calves were euthanized via captive bolt stunning and exsanguination, reticulo-rumens were harvested, emptied, and rinsed with cold water, then transported immediately to the laboratory for the following rumen dissection and tissue sampling procedure. The reticulo-rumen was placed on its left side, esophageal groove facing away, and an incision made around the circumference of the emptied and rinsed reticulo-rumen in line with the esophageal groove. A 6-cm section of the caudal portion of the caudal ventral blind sac was maintained intact. The rumen pillars were incised in line with the initial incision, and the muscles forming the rumen pillars separated. The reticulo-rumen was then opened and laid flat, creating a roughly symmetrical right and left side separated by the portion of the rumen maintained intact. The rumen pillars separate the rumen into 9 distinct sampling areas, shown in Figure 1 and labeled as (A) caudal portion of the caudal ventral blind sac; $(\mathbf{R B})$ right side and (LB) left side caudal dorsal sac; $(\mathbf{R C})$ right side and (LC) left side cranial dorsal sac; (RD) right side and

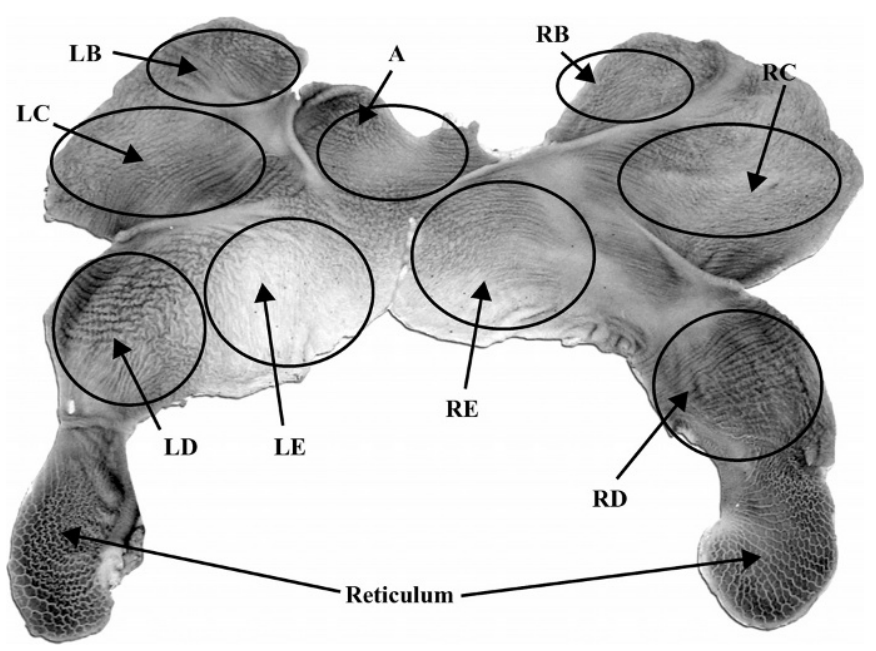

Figure 1. Example of a procedurally opened rumen depicting the physical areas of the rumen sampled and corresponding labels. (A) caudal portion of the caudal ventral blind sac; $(\mathrm{RB})$ right side and (LB) left side caudal dorsal sac; (RC) right side and (LC) left side cranial dorsal sac; (RD) right side and (LD) left side cranial ventral sac; and (RE) right side and (LE) left side ventral portion of caudal ventral blind sac.

(LD) left side cranial ventral sac; and (RE) right side and (LE) left side ventral portion of caudal ventral blind sac. A $1-\mathrm{cm}^{2}$ tissue sample was removed from the center of each area and four $1-\mathrm{cm}^{2}$ sections were removed $1 \mathrm{~cm}$ (for small areas or rumens) or $2 \mathrm{~cm}$ (for large areas or rumens) diagonally and distally from the 4 corners of the center sample $(n=5)$, creating a checkerboard pattern. Tissue samples were fixed in a $30 \%$ formaldehyde solution for subsequent measurements. Four randomly selected papillae per sample (20 per area) were measured for PL and PW, and one measurement per sample (5 per area) was recorded for RWT and PC. Visual measurements were taken using a Bausch and Lomb Stereo Zoom 4 dissecting microscope fitted with a measuring eyepiece at $11.25 \times$ magnification. Data from all 42 calves were pooled for procedure analysis.

\section{Statistical Analysis}

Correlations. Correlations for PL, PW, RWT, and PC between areas, samples, and measurements were obtained using the CORRELATION procedure of SAS (1999) and calculated as

$$
\rho_{i j}=\frac{\sigma_{i j}}{\sigma_{i} \sigma_{j}}
$$

where

$\rho_{\mathrm{ij}}=$ the correlation between PL, PW, RWT, or PC 
of the ith and jth areas, samples, or measurements;

$\sigma_{\mathrm{ij}}=$ the covariance of the ith and jth areas, samples, or measurements for PL, PW, RWT, or $\mathrm{PC}$;

$\sigma_{\mathrm{i}},\left(\sigma_{\mathrm{j}}\right)=$ the standard deviation of the ith (jth) area, sample, or measurement for PL, PW, RWT, or PC $\left(\sigma_{\mathrm{i}} \neq \sigma_{\mathrm{j}}\right)$; and

$\mathrm{i}, \mathrm{j}=\mathrm{A}, \mathrm{RB}, \mathrm{RC}, \mathrm{RD}, \mathrm{RE}, \mathrm{LB}, \mathrm{LC}, \mathrm{LD}$, or $\mathrm{LE}$ for area, 1 to 5 for sample, or 1 to 4 for measurement $(i \neq j)$.

Comparisons. Comparisons between experiments, areas, samples, and measurements for PL, PW, RWT, and PC were conducted across all calves and experiments using the MIXED procedure of SAS (1999) with a repeated measures statement. A separate model was utilized for PL and PW analysis than for RWT and PC analysis. The model for PL and PW analysis was

$$
\mathrm{y}_{\text {tasm }}=\mu+\alpha_{\mathrm{t}}+\beta_{\mathrm{a}}+\gamma_{\mathrm{s}}+\lambda_{\mathrm{m}}+\mathrm{e}_{\text {tasm }}
$$

where

$\mathrm{y}_{\text {tasm }}=$ an observed value for PL or PW for the mth measurement, taken from the sth sample, collected from the ath area from a calf in the tth experiment;

$\mu=$ the overall mean of the population;

$\alpha_{\mathrm{t}}=$ the fixed effect of the tth experiment where $\mathrm{t}=$ 1 to 4 ;

$\beta_{\mathrm{a}}=$ the random effect of the ath area where $\mathrm{a}=\mathrm{A}$, $\mathrm{RB}, \mathrm{RC}, \mathrm{RD}, \mathrm{RE}, \mathrm{LB}, \mathrm{LC}, \mathrm{LD}$, or LE;

$\gamma_{\mathrm{s}}=$ the random effect of the sth sample where $\mathrm{s}=$ 1 to 5 ;

$\lambda_{\mathrm{m}}=$ the random effect of the mth measurement where $\mathrm{m}=1$ to 4 ; and

$\mathrm{e}_{\text {tasm }}=$ the error associated with the mth measurement, taken from the sth sample, collected from the ath area from a calf in the th experiment; $e_{\text {tasm }} \approx N\left(0, \sigma_{e}^{2}\right)$.

Calf nested within experiment and sample nested within area were included in the RANDOM statement of the model, and measurement was utilized as the repeated variable. The model for RWT and PC analysis was

$$
\mathrm{y}_{\mathrm{tas}}=\mu+\alpha_{\mathrm{t}}+\beta_{\mathrm{a}}+\gamma_{\mathrm{s}}+\mathrm{e}_{\mathrm{tas}}
$$

where

$$
\begin{aligned}
y_{\text {tas }}= & \text { an observed value for RWT or PC for the sth } \\
& \text { sample, collected from the ath area from a calf } \\
& \text { in the th experiment; }
\end{aligned}
$$

$\mu=$ the overall mean of the population;

$\alpha_{\mathrm{t}}=$ the fixed effect of the th experiment where $\mathrm{t}=$ 1 to 4 ;

$\beta_{\mathrm{a}}=$ the random effect of the ath area where $\mathrm{a}=\mathrm{A}$, $\mathrm{RB}, \mathrm{RC}, \mathrm{RD}, \mathrm{RE}, \mathrm{LB}, \mathrm{LC}, \mathrm{LD}$, or LE;

$\gamma_{\mathrm{s}}=$ the random effect of the sth sample where $\mathrm{s}=$ 1 to 5 ; and

$\mathrm{e}_{\text {tas }}=$ the error associated with the sth sample, collected from the ath area from a calf in the th experiment; $e_{t a s} \approx N\left(0, \sigma_{e}^{2}\right)$.

Calf nested within experiment was included in the RANDOM statement, and sample was utilized as the repeated variable. Treatment influence was accounted for by including treatment in all comparison models. Age variation is accounted for by the inclusion of an experiment effect in the model. Differences between experiments were considered significant at $P<0.10$ and between areas, samples, or measurements at $P<0.001$. A strict $P$ value was utilized for area, sample, and measurement comparisons to insure that significant differences were also physiologically measurable differences.

Regression analysis. The REGRESSION procedure of SAS (1999) was utilized to determine relationships between PL, PW, RWT, and PC across all calves and experiments. Linear models were initially fit, subsequently followed by higher order models if applicable.

Power analysis. To determine the ability of the procedure to distinguish differences between rumen developmental levels, the necessary number of calves per treatment, samples per calf, measurements per calf, and the resultant power of each test, a power analysis was conducted for each described variable using a power macro (version 1.2) obtained from SAS (Latour, 2003). The power macro enabled the description of the desired $P$ value $(\boldsymbol{\alpha})$, root mean square error $(\boldsymbol{\sigma})$, and treatment difference or effect size $(\delta)$ calculated as $\delta=[\mathrm{SS}(\mathrm{Hyp}) /$ $\mathrm{n}]^{0.5}$ where

$\mathrm{SS}=$ sum of squares for the comparison of interest; Hyp = hypotheses stating that the effect size between 2 observed measurements for PL, PW, RWT, or $\mathrm{PC}$ equals 0 (null hypothesis) or that the effect size is not equal to 0 and is some nonzero number (alternate hypothesis); and

$\mathrm{n}=$ number of observations within the comparison of interest.

Effect size is determined by the difference between the observed effect size and the null hypothesis effect size. Levels for $\alpha$ were set at $0.01,0.05$, and 0.10 . Levels for $\sigma$ and $\delta$ were not defined by the researchers, but were calculated by the power macro through incorporation of 
the GLM procedure of SAS (1999) using the following model:

$$
\mathrm{y}_{\mathrm{pr}}=\mu+\alpha_{\mathrm{p}}+\beta_{\mathrm{r}}+\mathrm{e}_{\mathrm{pr}}
$$

where

$\mathrm{y}_{\mathrm{pr}}=$ an observed value for PL, PW, RWT, or PC from the rth calf, sample, or measurement in the pth rumen development group;

$\mu=$ the overall mean of the population;

$\alpha_{\mathrm{p}}=$ the fixed effect of the pth rumen development group where $\mathrm{p}=$ low or high;

$\beta_{\mathrm{r}}=$ the random effect of the rth calf, sample, or measurement where $r=1$ to 42 for calf; 1 to 5 for sample; or 1 to 4 for measurement; and

$\mathrm{e}_{\mathrm{pr}}=$ the error associated with the rth calf, sample, or measurement in the pth rumen development group; $e_{p r} \approx N\left(0, \sigma_{e}^{2}\right)$.

The power of the test at $\mathrm{n}$, the least significant number $(\mathbf{L S N})$, and the power of the test when $\mathrm{n}=\mathrm{LSN}$ were provided for each $\alpha$ level using the estimated $\sigma$ and $\delta$ values. The LSN is defined as the sample size required to produce a significant test for a sample having an $\alpha, \sigma$, and $\delta$ values equal to those of the data set used. Average values for PL, PW, RWT, and PC were calculated for each calf. The average values of PL, PW, and RWT were sorted from lowest to highest, with low average measurements considered as indicative of a low level of rumen development and high average measurements representative of a high level of rumen development. Average values for PC were sorted from highest to lowest with high PC values indicative of low rumen development (Anderson et al., 1982; Klein et al., 1987; Zitnan et al., 1999). The calves were then divided into 2 treatment groups, a low or high rumen development group, based on average variable measurements. Consideration was given to simply selecting the 21 lowest and highest averages and their corresponding calves, as this method would possibly decrease the observed variance for each rumen development variable. However, with the desire to make the results of this paper as realistic and applicable as possible, a selection criterion was developed. Division into treatment groups was as follows:

1) The 21 calves with the lowest average measurement for PL were marked as the low group, and the 21 calves with the highest average measurement for PL were marked as the high group.

2) For a calf to remain marked as low or high group required at least 2 average measurements for $\mathrm{PW}$, RWT, or PC within the 21 lowest or highest average measurements, respectively.
3) If a calf did not have 2 additional average measurements in the group corresponding with its PL, then an average measurement within the same group as PL was used to determine grouping. The order of importance for average measurements within the same group as PL was PW, RWT, and then PC.

Papillae length was the primary grouping factor due to analyses indicating a high level of correlation between PL throughout the rumen and stronger relationships between PL and the other 3 variables than between PW, RWT, or PC and the other 3 variables. The importance order in step 3 was developed using the same reasoning and statistics, with higher correlation values and stronger relationships for PW, followed by RWT, with PC having the lowest correlation values and weakest relationships. The power analysis step was conducted last to avoid any influence on previous analyses due to the selection criteria used. The power analyses utilized all 42 calves and included observations from all areas. Calves from all 4 experiments were present in both the low and high group accounting for experiment and age differences. All $\sigma$ and $\delta$ values presented were calculated by the power analyses and are unique to the data set utilized. Each calf, in the initial data set, had 180 observations for PL and PW (labeled 1 to 180) and 45 observations for RWT and PC (labeled 1 to 45) taken from 45 tissue samples (labeled 1 to 45) within the rumen. Average PL, PW, RWT, and PC were calculated for each calf to create the CALF power analysis data set. Average PL, PW, RWT, and PC were calculated for each sample ( 1 to 45 ) across calves within the given treatment group to create the SAMPLE power analysis dataset. For creation of the MEASUREMENT dataset, average PL and PW for each measurement (1 to 180) were calculated across calves within the treatment group. Data sets for SAMPLE and MEASUREMENT power analyses essentially represented 2 rumens, one with a high level of rumen development and one with a low level of rumen measurement, and contained 45 and 180 observations per treatment, respectively. Values averaged across calves within treatments were utilized due to a separate power analysis being conducted for each effect of interest (calf, sample, and measurement); therefore each effect analyzed was considered a main effect within their respective power analysis (Dawson and Lagakos, 1993). Calves within treatment and observations within calves were the same for all data sets. Variation attributable to each of the dependent variables of interest (i.e., calves, samples, measurements) was captured by the manner in which averages were calculated for each distinct data set, and the number of observations within each data set. 
Table 1. Least square means for papillae length (PL), papillae width (PW), rumen wall thickness (RWT), and papillae per $\mathrm{cm}^{2}(\mathrm{PC})$ for different experiments.

\begin{tabular}{lllllllllll}
\hline & & \multicolumn{7}{c}{ Least square means and SEM for rumen variables } \\
\cline { 5 - 10 } Experiment & $\mathrm{n}$ & age, wk & PL, mm & SEM & PW, mm & SEM & RWT, mm & SEM & PC & SEM \\
\hline 1 & 6 & 6 & $1.30^{\mathrm{a}}$ & 0.26 & $0.82^{\mathrm{a}}$ & 0.13 & $1.48^{\mathrm{a}}$ & 0.10 & $86.37^{\mathrm{a}}$ & 9.36 \\
2 & 6 & 5 & $1.22^{\mathrm{ab}}$ & 0.26 & $0.72^{\mathrm{ab}}$ & 0.13 & $1.51^{\mathrm{a}}$ & 0.10 & $70.82^{\mathrm{b}}$ & 9.36 \\
3 & 15 & 4 & $0.81^{\mathrm{b}}$ & 0.09 & $0.55^{\mathrm{b}}$ & 0.05 & $1.12^{\mathrm{b}}$ & 0.04 & $75.98^{\mathrm{ab}}$ & 3.30 \\
4 & 15 & 5 & $1.10^{\mathrm{ab}}$ & 0.21 & $0.72^{\mathrm{ab}}$ & 0.10 & $1.40^{\mathrm{a}}$ & 0.08 & $70.27^{\mathrm{ab}}$ & 7.52 \\
\hline \multicolumn{2}{c}{${ }^{\mathrm{a}, \mathrm{b}}$ Means within a column without common }
\end{tabular}

${ }^{, b}$ Means within a column without common superscripts are different with a $P<0.10$.

\section{RESULTS}

\section{Experiment Analysis}

Values for least square means, standard error, and comparisons for PL, PW, RWT, and PC from the 4 experiments are presented in Table 1 . Values appear to be influenced by age across experiments with significant differences $(P<0.10)$ occurring between experiment 3 and experiment 1 for PL and PW, between experiment 3 and all other experiments for RWT, and between experiment 2 and experiment 1 for PC. Rumens from experiment 3 were collected from calves at 4 wk of age and represent the youngest group of animals in the data set; therefore, lower values for PL, PW, and RWT were not unexpected. However, similar values between samples obtained at 5 and 6 wk of age were not expected and may indicate a rapidly developing rumen prior to $5 \mathrm{wk}$ of age, with a slower rate of development after $5 \mathrm{wk}$ of age, under the calf rearing conditions utilized.

\section{Area Analysis}

Correlations. Correlation values for PL and PW between areas were relatively high in all cases, ranging from 0.42 to 0.67 for PL and 0.38 to 0.60 for PW. All correlation values for PL and PW were significantly different $(P<0.001)$ from 0 . Correlations for RWT and PC between areas were not as strong and were highly variable with values ranging from 0.18 to 0.49 for RWT and -0.06 to 0.44 for PC. All correlation values for RWT were significantly different $(P<0.05)$ from 0 , but not as consistent as observed for PL and PW. Correlation values for PC were not all significantly different from 0 .

Comparisons. Values for least square means, standard error, and comparisons of PL, PW, RWT, and PC for areas across calves and experiments are presented in Table 2. Significant differences $(P<0.001)$ were observed between some areas for PL, PW, RWT, and PC. Occurrences of difference between areas may be primarily explained by intrarumen variation. In addition, standard errors for PL and PW made up a larger portion of the mean than standard errors for RWT and PC, indicating greater variation within the former rumen development parameters. Similar values and minimal significant differences between areas corresponding to the caudal portion of the caudal ventral blind sac and the caudal dorsal sac (A and B) were observed, indicating that samples from either area could represent the caudal rumen. $\mathrm{Nu}-$ merous differences between right and left rumen areas were observed for PL, with only one difference observed for PW and RWT, and no right and left rumen area differences seen for PC. More differences between right and left rumen areas for PL may be explained by higher variation within $\mathrm{PL}$ when compared with $\mathrm{PW}$, RWT, and PC.

Table 2. Least square means for papillae length (PL), papillae width (PW), rumen wall thickness (RWT), and papillae per $\mathrm{cm}^{2}$ (PC) for different areas of the rumen across all calves and experiments.

\begin{tabular}{|c|c|c|c|c|c|c|c|c|}
\hline \multirow[b]{2}{*}{ Area } & \multicolumn{8}{|c|}{ Least square means and SEM for rumen variables } \\
\hline & $\mathrm{PL}, \mathrm{mm}$ & SEM & PW, mm & SEM & RWT, mm & SEM & $\mathrm{PC}$ & SEM \\
\hline $\mathrm{A}$ & $1.09^{\mathrm{a}}$ & 0.09 & $0.74^{\mathrm{ab}}$ & 0.05 & $1.24^{\mathrm{a}}$ & 0.04 & $77.89^{\text {acd }}$ & 3.40 \\
\hline $\mathrm{RB}$ & $1.13^{\mathrm{a}}$ & 0.09 & $0.73^{\mathrm{ab}}$ & 0.05 & $1.24^{\mathrm{a}}$ & 0.04 & $78.41^{\text {acd }}$ & 3.40 \\
\hline $\mathrm{RC}$ & $0.97^{\mathrm{b}}$ & 0.09 & $0.72^{\mathrm{a}}$ & 0.05 & $1.34^{\mathrm{b}}$ & 0.04 & $71.88^{\text {bd }}$ & 3.40 \\
\hline $\mathrm{RD}$ & $2.01^{\mathrm{c}}$ & 0.09 & $0.81^{\mathrm{c}}$ & 0.05 & $1.63^{\mathrm{c}}$ & 0.04 & $67.46^{\mathrm{bd}}$ & 3.40 \\
\hline $\mathrm{RE}$ & $0.63^{\mathrm{d}}$ & 0.09 & $0.58^{\mathrm{d}}$ & 0.05 & $1.30^{\mathrm{abd}}$ & 0.04 & $79.98^{\mathrm{ce}}$ & 3.40 \\
\hline LB & $1.09^{\mathrm{a}}$ & 0.09 & $0.72^{\mathrm{a}}$ & 0.05 & $1.26^{\text {abe }}$ & 0.04 & $77.93^{\text {cde }}$ & 3.40 \\
\hline $\mathrm{LC}$ & $1.14^{\mathrm{a}}$ & 0.09 & $0.76^{\text {ae }}$ & 0.05 & $1.44^{\mathrm{e}}$ & 0.04 & $72.51^{\mathrm{bd}}$ & 3.40 \\
\hline LD & $1.43^{\mathrm{e}}$ & 0.09 & $0.77^{\text {bce }}$ & 0.05 & $1.61^{\mathrm{c}}$ & 0.04 & $72.08^{\text {bd }}$ & 3.40 \\
\hline LE & $0.48^{\mathrm{f}}$ & 0.09 & $0.49^{f}$ & 0.05 & $1.35^{\text {de }}$ & 0.04 & $84.60^{\mathrm{e}}$ & 3.40 \\
\hline
\end{tabular}




\section{Sample and Measurement Analysis}

Correlations. All correlation values for variables PL, PW, RWT, and PC between different samples across areas, calves, and experiments were consistently high and significantly different $(P<0.001)$ from 0 , showing a strong correlation between samples for these variables. Correlation values range from 0.70 to 0.77 for PL, 0.57 to 0.61 for PW, 0.44 to 0.58 for RWT, and from 0.55 to 0.65 for PC.

Similar correlation results were observed between measurements taken across samples, areas, calves, and experiments. Measurement correlation values for variables PL and PW were all significantly different $(P<$ 0.001 ) from 0 , and ranged from 0.90 to 0.92 for PL and from 0.72 to 0.76 for $\mathrm{PW}$, indicating a strong correlation between measurements for PL and PW.

Comparisons. Values for least square means of PL (average $1.11 \mathrm{~mm}$ ), PW (average $0.70 \mathrm{~mm}$ ), RWT (average $1.38 \mathrm{~mm}$ ), and PC (average 75.86) were similar for different samples across areas, calves, and experiments with no significant differences between samples. Standard errors as a percentage of means were highest for variable PL (0.09) followed by PW (0.05), PC (3.30), then RWT (0.04), as observed in the area analysis.

No significant differences were observed between least square means of PL (average $1.11 \mathrm{~mm}$ ) and PW (average $0.70 \mathrm{~mm}$ ) from measurements taken across samples, areas, calves, and experiments. Standard errors as a percentage of means were once again highest for variable PL (0.09) followed by PW (0.04), as seen in the area and sample analyses.

\section{Rumen Variable Relationships}

All calves and experiments were included in the regression analyses. Relationships between most rumen variable pairs were minimal, except for PL and PW. There was a significant linear and quadratic relationship between PL and PW. The linear regression was:

$$
\begin{gathered}
\mathrm{PL}=-0.05( \pm 0.02) \\
+1.62( \pm 0.03) \mathrm{PW} \text { with } \mathrm{R}^{2}=0.58
\end{gathered}
$$

The quadratic relationships between PL and PW were:

$$
\begin{gathered}
\mathrm{PL}=-0.11( \pm 0.04)+1.85( \pm 0.12) \mathrm{PW} \\
-0.17( \pm 0.09) \mathrm{PW}^{2} \text { with } \mathrm{R}^{2}=0.58 \text { and } \\
\mathrm{PW}=1.16( \pm 0.01)+0.69( \pm 0.02) \mathrm{PL} \\
-0.15( \pm 0.01) \mathrm{PL}^{2} \text { with } \mathrm{R}^{2}=0.63
\end{gathered}
$$

\section{Power Analysis}

Calves, samples, and measurements required. Table 3 presents the results of power analyses conducted to determine the number of calves, samples, and measurements necessary to find significant differences between treatments with an $\alpha$ of $0.01,0.05$, or 0.10 for PL, PW, RWT, and PC. Results of the power analysis for PL, $\mathrm{PW}$, and RWT indicate the ability of the procedure to detect treatment differences, at an acceptably high power, with a data set containing 21 calves per treatment, 45 samples, and 180 measurements per calf. Results of the power analysis for PC indicate an inability of the procedure to detect significant differences between treatments with the number of samples utilized.

LSN and power when $\boldsymbol{N}=\boldsymbol{L S N}$. Values for LSN indicate the total number of calves, samples per calf, and measurements per calf necessary to detect treatment difference. As 2 treatments were utilized in the analyses, the value for LSN should be divided by 2 to determine the lowest number of calves per treatment, samples per calf, and measurements per calf necessary to detect treatment differences. However, the power of the test when $\mathrm{n}=$ LSN may not be sufficiently high to warrant acceptability. Therefore, values for LSN should be considered threshold numbers to detect difference. In addition, as the observed $\sigma, \delta$, and LSN values are unique to this data set, LSN values may not be universally applicable, but do provide a starting point for research design and planning. With the observed $\alpha, \sigma$, and $\delta$ values, the results from Table 3 suggest 5,3 , or 3 calves per treatment, 5, 3, or 3 samples per calf, and 9, 6 , or 4 measurements per calf to detect treatment differences for PL with an $\alpha$ of $0.01,0.05$, and 0.10 , respectively. With the same format, the results suggest 6,4 , or 3 calves per treatment, 3,3 , or 2 samples per calf, and 5,3 , or 3 measurements per calf to detect treatment difference for PW. Results for RWT suggest 18, 11, or 8 calves per treatment and 13,8 , or 6 samples/measurements per calf to detect treatment differences with an $\alpha$ of $0.01,0.05$, or 0.10 , respectively. Values of LSN for RWT are higher than for PL and PW, possibly due to low $\delta$ values (observed difference between treatments) for this variable. Due to low $\delta$ values and low relationship between $\delta$ and $\sigma$, values of LSN for PC are high, suggesting that $\mathrm{PC}$ may not be a feasible variable in rumen development research.

\section{DISCUSSION}

\section{Correlations}

Strong area, sample, and measurement correlations suggest a high probability that a change in PL, PW, RWT, or PC from any area, sample, or measurement will reflect a similar change for any other area, sample, or measurement, more with PL, PW, and RWT than PC. This indicates the possibility that samples taken from some areas of the rumen will represent rumen develop- 
Table 3. Results of power analysis for number of calves, samples, and measurements required for papillae length, papillae width, rumen wall thickness, and papillae per $\mathrm{cm}^{2}$.

\begin{tabular}{|c|c|c|c|c|c|c|c|}
\hline$\underline{\text { Rumen variable }}$ & $\alpha^{1}$ & $\mathrm{n}$ & $\sigma^{1}$ & $\delta^{1}$ & $\begin{array}{l}\text { Power } \\
\text { at } n\end{array}$ & $\mathrm{LSN}^{1}$ & $\begin{array}{l}\text { Power } \\
\mathrm{n}=\mathrm{LSN}\end{array}$ \\
\hline \multicolumn{8}{|l|}{ Calves required } \\
\hline \multirow[t]{3}{*}{ Papillae length } & 0.01 & 42 & 0.25 & 0.31 & 1.00 & 9 & 0.59 \\
\hline & 0.05 & 42 & 0.25 & 0.31 & 1.00 & 6 & 0.62 \\
\hline & 0.10 & 42 & 0.25 & 0.31 & 1.00 & 5 & 0.67 \\
\hline \multirow[t]{3}{*}{ Papillae width } & 0.01 & 42 & 0.14 & 0.14 & 1.00 & 11 & 0.55 \\
\hline & 0.05 & 42 & 0.14 & 0.14 & 1.00 & 7 & 0.57 \\
\hline & 0.10 & 42 & 0.14 & 0.14 & 1.00 & 6 & 0.64 \\
\hline \multirow[t]{3}{*}{ Rumen wall thickness } & 0.01 & 42 & 0.19 & 0.09 & 0.63 & 35 & 0.52 \\
\hline & 0.05 & 42 & 0.19 & 0.09 & 0.84 & 21 & 0.53 \\
\hline & 0.10 & 42 & 0.19 & 0.09 & 0.91 & 15 & 0.53 \\
\hline \multirow[t]{3}{*}{ Papillae per $\mathrm{cm}^{2}$} & 0.01 & 42 & 12.96 & 0.81 & 0.02 & 1688 & 0.50 \\
\hline & 0.05 & 42 & 12.96 & 0.81 & 0.07 & 978 & 0.50 \\
\hline & 0.10 & 42 & 12.96 & 0.81 & 0.13 & 686 & 0.50 \\
\hline \multicolumn{8}{|l|}{ Samples required } \\
\hline \multirow{3}{*}{ Papillae length } & 0.01 & 90 & 0.25 & 0.31 & 1.00 & 9 & 0.59 \\
\hline & 0.05 & 90 & 0.25 & 0.31 & 1.00 & 6 & 0.62 \\
\hline & 0.10 & 90 & 0.25 & 0.31 & 1.00 & 5 & 0.67 \\
\hline \multirow[t]{3}{*}{ Papillae width } & 0.01 & 90 & 0.07 & 0.14 & 1.00 & 6 & 0.63 \\
\hline & 0.05 & 90 & 0.07 & 0.14 & 1.00 & 5 & 0.83 \\
\hline & 0.10 & 90 & 0.07 & 0.14 & 1.00 & 4 & 0.80 \\
\hline \multirow[t]{3}{*}{ Rumen wall thickness } & 0.01 & 90 & 0.16 & 0.09 & 1.00 & 25 & 0.53 \\
\hline & 0.05 & 90 & 0.16 & 0.09 & 1.00 & 15 & 0.53 \\
\hline & 0.10 & 90 & 0.16 & 0.09 & 1.00 & 11 & 0.54 \\
\hline \multirow[t]{3}{*}{ Papillae per $\mathrm{cm}^{2}$} & 0.01 & 90 & 6.11 & 1.13 & 0.20 & 198 & 0.50 \\
\hline & 0.05 & 90 & 6.11 & 1.13 & 0.41 & 115 & 0.50 \\
\hline & 0.10 & 90 & 6.11 & 1.13 & 0.54 & 81 & 0.50 \\
\hline \multicolumn{8}{|l|}{ Measurements required $^{2}$} \\
\hline \multirow[t]{3}{*}{ Papillae length } & 0.01 & 360 & 0.44 & 0.30 & 1.00 & 18 & 0.52 \\
\hline & 0.05 & 360 & 0.44 & 0.30 & 1.00 & 11 & 0.54 \\
\hline & 0.10 & 360 & 0.44 & 0.30 & 1.00 & 8 & 0.54 \\
\hline \multirow[t]{3}{*}{ Papillae width } & 0.01 & 360 & 0.11 & 0.14 & 1.00 & 9 & 0.62 \\
\hline & 0.05 & 360 & 0.11 & 0.14 & 1.00 & 6 & 0.64 \\
\hline & 0.10 & 360 & 0.11 & 0.14 & 1.00 & 5 & 0.69 \\
\hline
\end{tabular}

${ }^{1} \alpha=$ Desired $P$ value; $\sigma=$ root mean square error; $\delta=$ treatment difference; LSN = lowest necessary number to detect significance; to determine LSN per treatment, values should be divided by 2 .

${ }^{2}$ Multiple measurements for rumen wall thickness or papillae per $\mathrm{cm}^{2}$ were not conducted.

ment as effectively as samples taken from all areas of the rumen. In addition, results also indicated high similarity between multiple samples and/or measurements taken from the same area, suggesting the possibility for reduced samples and/or measurements. Correlations between similar areas on the right and left sides of the rumen were also high, indicating that samples from either side could be representative of that entire area of the rumen. In addition, similarities between areas A, $\mathrm{RB}$, and LB suggest that samples taken from any one of these areas will be representative of caudal rumen development. As stated, previous research has commonly obtained rumen tissue samples from the caudal dorsal and/or cranial ventral sacs of the rumen, which correspond to areas $\mathrm{RB}, \mathrm{LB}, \mathrm{RD}$, and $\mathrm{LD}$, respectively, indicating results from previous research to be sufficiently representative of rumen development (Tamate et al., 1962; Anderson et al., 1982; Nocek et al., 1984). Representation of area E (caudal ventral blind sac) is limited in previous research. Lack of representation of area $\mathrm{E}$ is not surprising, as it appears that this area is less developed in the young ruminant calf. However, inclusion of area E, even if less developed, may aid in distinguishing treatment effects on development of the entire rumen.

\section{Rumen Variable Relationships}

The relationship among and explained variation of the rumen variables is greatest for $\mathrm{PL}$, followed by $\mathrm{PW}$, then RWT, with PC having little to no relationship with the other variables. In addition, the regression of PL, PW, and RWT on PC indicated a slight negative relationship. This suggests that as PC increases, values for PL, PW, and RWT decrease, a trait reported in previous research (Anderson et al., 1982; Klein et al., 1987; Zitnan et al., 1999). This relationship is not surprising as increased values for PL, PW, and RWT are indicative of increased rumen and papillary growth and development. An increase in the size of papillae in a fixed $1-\mathrm{cm}^{2}$ section of 
the rumen wall must result in a decrease in the number of papillae in that finite area. In addition, an increased rumen volume, possibly represented by an increased RWT, enlarges the area covered by $1-\mathrm{cm}^{2}$ at birth over a larger area at a later age, thereby decreasing the number of papillae in the finite section once again.

\section{Comparison and Power Analyses}

Papillae length. Values for PL indicate a greater occurrence of treatment and area differences and high variability within $P L$. The high variation and differences may explain the increased power of the procedure to detect treatment differences in PL, as presented in Table 3. Therefore, PL may be the most important variable for rumen development research, and may represent the greatest influence of treatment on rumen development. However, high PL variability may also increase the number of areas that require sampling to sufficiently represent development of the entire rumen. Previous research has detected treatment differences for PL when weaning date or the dry portion of the ration was chemically or physically altered (Nocek et al., 1984; Greenwood et al., 1997; Zitnan et al., 1999). These previous findings are expected as rumen development is greatly influenced by age and the presence of butyrate from microbial and protozoal degradation of readily fermentable carbohydrate sources in the rumen (Brownlee, 1956; Warner et al., 1956; Klein et al., 1987). Other research has not detected treatment differences in PL, but has detected age differences (Anderson et al., 1982; Klein et al., 1987).

Papillae width. Values for PW indicate a lower occurrence of treatment and area differences and decreased variability within PW than PL, but higher than RWT and PC. These values possibly explain why the power of PW falls between the power for PL and RWT (Table 3). However, the power of the procedure to detect treatment differences for PW is also very high. Therefore, these results suggest that PW is likely a secondarily important rumen variable for rumen development research. Detection of differences in PW has been limited in previous research, and weaning age appears to have a greater effect than chemical or physical alteration of the dry ration (Klein et al., 1987; Greenwood et al., 1997; Zitnan et al., 1999).

Rumen wall thickness. It appears that RWT is tertiary in importance as a rumen development variable. Statistical treatment differences can be obtained with the procedure, but at a decreased ability than indicated for PL and PW, possibly due to insufficient observations per area or the inability of treatment differences to influence this variable to the same extent as PL and PW. Previous research has not detected significant differences between treatments for rumen wall and/or epithe- lial thickness when measurements were obtained in areas similar to those described for this procedure (Anderson et al., 1982; Greenwood et al., 1997). In addition, it has been suggested that an increase in rumen muscularization may occur independently of rumen epithelial growth (Brownlee, 1956; Harrison et al., 1960). However, as no attempts were made to separate the rumen muscle from the rumen epithelium in this procedure, the results in these analyses may be confounded.

Papillae per square centimeter. Results for PC indicate a low power, or possible inability, of the procedure to detect treatment differences in PC, suggesting inapplicability of PC as a rumen development variable. The inability of the procedure to detect treatment differences is likely a result of high $\sigma$ values and low $\delta$ values. It is also possible that the described procedure is incapable of detecting treatment differences for $\mathrm{PC}$ due to sampling error. In addition, the possibility of high genetic control over PC, subsequently limiting environmental influence, should also be considered. Age of the calf, age at weaning, and subsequent length of time that concentrates make up an appreciable portion of the daily ration are likely the environmental factors having the greatest influence on detectable differences in PC and therefore have the highest possibility of confounding results (Klein et al., 1987; Zitnan et al., 1998; Zitnan et al., 1999). Furthermore, age of the calf and related rumen volume has a tremendous effect on the finite area of the rumen sampled, as described in the rumen variable relationships section. However, some researchers have reported significant differences between treatments for this variable (Anderson et al., 1982; Nocek et al., 1984).

\section{Calf Numbers, Samples, and Measurements}

It is apparent from the results that the areas sampled, the number of tissue samples taken, and the number of measurements per area can be reduced from that originally suggested by this procedure. Papillae length is the only variable appearing to require sampling from the right and left side of the rumen. However, a high power of the test for PL may overcome the need for right and left side representation. In addition, the occurrence of only one right and left side difference for PW and RWT, and no differences for PC, coupled with relatively high correlations between right and left rumen areas, indicates a limited need for sampling both the right and left side of the rumen. Therefore, the rumen dissection procedure reported by McGavin and Morrill (1976a) may be effective for dissecting and sampling the reticulo-rumen. However, the visible guides utilized for dissection may not be readily apparent in the young calf, increasing the difficulty of the McGavin and Morrill (1976a) dissecting procedure. Due to the results indicated, it is rec- 
ommended that tissue samples be obtained from the cranial and caudal sacs of the ventral and dorsal rumen (area $\mathrm{n}=4$ ), 3 random tissue samples collected from each area (sample $\mathrm{n}=12$ ), and 2 measurements per sample for PL, PW, and RWT recorded (measurement $\mathrm{n}=24$ ). For purposes of representing physiological growth throughout the entire rumen, multiple sampling sites are suggested. However, for statistical purposes, samples collected from a specific rumen area may also be acceptable, provided that rumen sampling site is identical across calves and treatments. Twenty-four measurements per calf reduces the originally suggested sample numbers by $87 \%$ for PL and PW and by $47 \%$ for RWT, and should result in statistically acceptable power for PL and PW, but may be limited in statistical power for RWT. Therefore, more RWT measurements per calf may be desirable but will require additional tissue samples. However, the possible inapplicability of RWT may not warrant additional time spent on this variable, and PL or PW may sufficiently address rumen epithelial changes. It is not suggested to record PC measurements due to the high numbers required to find statistical differences for this variable. The required calves per treatment to find differences range from 3 to 18; however, these values result in detectable treatment difference with a possibly unacceptable power. In addition, it is not suggested to use less than 3 calves per treatment to avoid outlier influence and maintain degrees of freedom within treatment. It appears that 3 calves per treatment should result in an analysis of sufficient power for PL and PW, valid indicators of rumen development, and minimally limited power for RWT.

\section{CONCLUSION}

This rumen sampling procedure appears to have sufficient ability to detect treatment differences for PL and PW, with reduced, but possibly acceptable, ability for RWT. In contrast, there appears to be limited to no ability for detecting differences in PC, possibly due to inadequate observations, high variability, or a decreased treatment influence due to high genetic influence. In addition, the degree of variability for PL and PW appears to be relatively high, even with a data set of this size. Conversely, variability for RWT and PC is lower when compared to PL and PW, and may indicate decreased ability to influence the former parameters. It is hoped that the procedure and information presented in this paper will provide a means to increase the comparability of future rumen development research and aid in the understanding of treatment effects on rumen development in young dairy calves.

\section{ACKNOWLEDGMENTS}

This research was a component of NC-1119, Management Systems to Improve the Economic and Environmental Sustainability of Dairy Enterprises. In addition, the authors extend their appreciation to Alltech, Inc., Nicholasville, KY, for partial funding of this research.

\section{REFERENCES}

Anderson, M. J., M. Khoyloo, and J. L. Walters. 1982. Effect of feeding whole cottonseed on intake, body weight, and reticulorumen development of young Holstein calves. J. Dairy Sci. 65:764-772.

Brownlee, A. 1956. The development of rumen papillae in cattle fed on different diets. Br. Vet. J. 112:369-375.

Dawson, J. D., and S. W. Lagakos. 1993. Size and power of two-sample tests of repeated measures data. Biometrics 49:1022-1032.

Flatt, W. P., R. G. Warner, and J. K. Loosli. 1958. Influence of purified materials on the development of the ruminant stomach. J. Dairy Sci. 41:1593-1600.

Greenwood, R. H., J. L. Morrill, E. C. Titgemeyer, and G. A. Kennedy. 1997. A new method of measuring diet abrasion and its effect on the development of the forestomach. J. Dairy Sci. 80:2534-2541.

Harrison, H. N., R. G. Warner, E. G. Sander, and J. K Loosli. 1960. Changes in the tissue and volume of the stomachs of calves following removal of dry feed or consumption of inert bulk. J. Dairy Sci. 43:1301-1312.

Klein, R. D., R. L. Kincaid, A. S. Hodgson, J. H. Harrison, J. K. Hillers, and J. D. Cronrath. 1987. Dietary fiber and early weaning on growth and rumen development of calves. J. Dairy Sci. 70:20952104

Latour, K. R. 2003. \%Power: A simple macro for power and sample size calculations. http://support.sas.com/techsup/technote/ ts272.pdf. Accessed May 20, 2003.

McGavin, M. D., and J. L. Morrill. 1976a. Dissection technique for examination of the bovine ruminoreticulum. J. Anim. Sci. 42:535-538.

McGavin, M. D., and J. L. Morrill. 1976b. Scanning electron microscopy of ruminal papillae in calves fed various amount and forms of roughage. Am. J. Vet. Res. 37:497-508.

Nocek, J. E., C. W. Heald, and C. E. Polan. 1984. Influence of ration physical form and nitrogen availability on ruminal morphology of growing bull calves. J. Dairy Sci. 67:334-343.

SAS. 1999. SAS/STAT User's Guide (Version 8.01 Edition). SAS Inst. Inc., Cary, NC.

Sutton, J. D., A. D. McGilliard, and N. L. Jacobson. 1963. Functional development of rumen mucosa. I. Absorptive ability. J. Dairy Sci. 46:426-436.

Tamate, H., A. D. McGilliard, N. L. Jacobson, and R. Getty. 1962. Effect of various dietaries on the anatomical development of the stomach in the calf. J. Dairy Sci. 45:408-420.

Van Soest, P. J. 1994. Nutritional Ecology of the Ruminant. 2nd ed. Cornell University Press, Ithaca, NY.

Warner, R. G., W. P. Flatt, and J. K. Loosli. 1956. Dietary factors influencing the development of the ruminant stomach. J. Agric. Food Chem. 4:788-792.

Zitnan, R., J. Voigt, U. Schonhusen, J. Wegner, M. Kokardova, H. Hagemeister, M. Levkut, S. Kuhla, and A. Sommer. 1998. Influence of dietary concentrate to forage ratio on the development of rumen mucosa in calves. Arch. Anim. Nutr. 51:279-291.

Zitnan, R., J. Voigt, J. Wegner, G. Breves, B. Schroder, C. Winckler, M. Levkut, M. Kokardova, U. Schonhusen, S. Kuhla, H. Hagemeister, and A. Sommer. 1999. Morphological and functional development of the rumen in the calf: Influence of the time of weaning. Arch. Anim. Nutr. 52:351-362. 